\title{
Abstracts der Jahrestagung der Österreichischen Gesellschaft für Senologie (27.-29.09.18, Graz)
}

01

Brustrekonstruktion mit Implantaten:

Einfluss der postoperativen Strahlentherapie auf das kosmetische Ergebnis

Sokullu, F.; Zeyghami, A.; Lewicki, M.; Özdemir, Ö.; Kömürcü, F.; Deutinger, M. Abteilung für Plastische Chirurgie, Krankenanstalt Rudolfstiftung, Wien

Keywords: Brustrekonstruktion, Bestrahlung, Implantate, Komplikationen, Mammakarzinom

Kategorie: Oral presentation or poster

Hintergrund: Ziel jeder Brustkrebsbehandlung ist es, Mammakarzinompatientinnen so schonend wie möglich $\mathrm{zu}$ therapieren. Sollte eine brusterhaltende Therapie nicht möglich sein, so können im Rahmen der Mastektomie, skin-sparing Mastektomie oder skin und nipple-sparing Mastektomie verschiedene operative Verfahren der Brustrekonstruktion eingesetzt werden. $\mathrm{Zu}$ den alloplastischen Methoden zählen die primäre Implantatrekonstruktion und die primäre und sekundäre ExpanderImplantat-Rekonstruktion. Ist eine adjuvante Strahlentherapie indiziert, führt es oft zu unzufriedenen kosmetischen Ergebnissen. Ziel dieser retrospektiven Studie war es herauszufinden inwiefern sich die Strahlentherapie auf das kosmetische Ergebnis auswirkt. Zusätzlich wurde die Auswirkung der Strahlentherapie auf die Komplikationsrate analysiert.

Methoden: Von insgesamt 37 Mammakarzinompatientinnen nach Mastektomie und Sofortrekonstruktion mit Implantaten, erfolgte bei $10 \mathrm{~Pa}$ tientinnen eine adjuvante Bestrahlung. Im Rahmen einer retrospektiven Analyse wurde das kosmetische Ergebnis zwischen den 2 Gruppen verglichen. Zusätzlich wurde mittels des modified health questionnaire SF - 36 die Lebensqualität der Patientinnen der 2 Gruppen evaluiert.

Ergebnisse: Die bestrahlte Gruppe entwickelte deutlich mehr Komplikationen (z.B. Kapselfibrose, Infektionen, Serome, Wundheilungsstörungen,...) im Vergleich zur nicht bestrahlten Gruppe. Ein Unterschied in der Lebensqualität ergab kein signifikantes Ergebnis, jedoch gab es eine höhere Verbesserung der Lebensqualität in der nicht bestrahlten Gruppe. Zusammenfassung: In der Literatur wird die adjuvante Strahlentherapie als die wichtigste relative Kontraindikation zur Implantatrekonstruktion beschrieben. Diese führt zu einem Anstieg der Komplikationsrate, weswegen bei Mammakarzinompatientinnen, bei denen eine adjuvante Bestrahlung notwendig ist, eine ausführliche Aufklärung über die Risiken erfolgen sollte.
02 Freier Vortrag

Ergebnisse der Mammadatenbank des BGZ Wilhelminenspital

Abayev, S.; Schlenz, I.; Koller, R.

Plastische Chirurgie, Wilhelminenspital, Wien

Keywords: BGZ Wilhelminenspital, Rekonstruktionsmethoden, Methodenvergleich, Komplikationen, Erfolgsrate

Topic: update Mammachirurgie

Die Brustrekonstruktion hat einen wichtigen Stellenwert in der Behandlung des Mammakarzinoms. Durch die Weiterentwicklung der Rekonstruktionsmethoden können in den letzten Jahren den Patientinnen vermehrt Sofortrekonstruktionen angeboten werden, was sowohl das ästhetische als auch das psychische Outcome verbessert. Die Entscheidung, ob Eigengewebe oder Prothesen für die Rekonstruktion verwendet werden, hängt oft von den Möglichkeiten des behandelnden Zentrums ab. In der folgenden Studie soll veranschaulicht werden, welches Spektrum im BGZ Wilhelminenspital den Patientinnen angeboten wird und wie die Ergebnisse der einzelnen Rekonstruktionsmethoden im Vergleich abschneiden. In den Jahren 2003-2018 wurden am BGZ Wilhelminenspital 835 Mammarekonstruktionen durchgeführt, davon 61\% Sofort- und 39\% Sekundärrekonstruktionen. In $42,8 \%$ handelte es sich um reine Implantatrekonstruktionen, in 57,2\% kam Eigengewebe zum Einsatz. Die Misserfolgsrate bei Implantatrekonstruktionen betrug 4,1\%, bei Eigengewebe $0,9 \%(p=0,023)$. Die Komplikationen bei Implantaten betragen in der überwiegenden Zahl DTI Fälle (36,7\%), lediglich 17 (19,1\%) Expander mussten entfernt werden.

Wie in anderen Studien sind Rauchen, Vorbestrahlung und Adipositas als wesentliche Risikofaktoren anzusehen. Eigengewebemethoden machen längerfristig die risikoärmere Rekonstruktionsmethode aus. Bei sorgfältiger Planung unter Berücksichtigung der Risikofaktoren, des Patientinnenwunsches und der möglichen Rekonstruktionsverfahren können gute Ergebnisse mit geringem Komplikationsrisiko erzielt werden.

03

Prophylaktische Mastektomie und Brustrekonstruktion bei Hochrisikopatientinnen - Ergebnisse des BGZ Wilhelminenspital

Koller, R. ${ }^{1}$; Abayev, S. ${ }^{1}$; Stocker, B. ${ }^{2}$; Bartl, M. ${ }^{3}$

${ }^{1}$ Abteilung für Plastische Chirurgie, Wilhelminenspital, Wien

${ }^{2}$ Abteilung für Chirurgie, Wilhelminenspital, Wien

${ }^{2}$ Abteilung für Gynäkologie - Geburtshilfe, Wilhelminenspital, Wien

Keywords: Risikopatientinnen, Komplikationen, prophylaktische Mastektomie, Aufklärung, DTI

\section{KARGER}

(c) 2018 S. Karger GmbH, Freiburg 
Topic: prophylaktische Mastektomie

Die vermehrte Aufklärung und Aufmerksamkeit auf familiäres Hochrisiko für Brustkrebserkrankungen und die Möglichkeit der Testung auf verschiedene Genmutationen sowie die Möglichkeit der Brustwarzen-erhaltenden Operation lässt die Anzahl der risikoreduzierenden Mastektomien steigen. Während es in den USA den Trend gibt, den alleinigen Wunsch der Patientin als Anlass zu nehmen, eine Brustentfernung durchzuführen, hat sich in Europa der Standard der vorherigen Testung auf Genmutationen etabliert. Die folgende Studie zeigt die Ergebnisse nach Brustrekonstruktion bei Hochrisikopatientinnen verglichen mit Patientinnen ohne bekanntem Risiko und setzt sich kritisch mit den Resultaten auseinander.

Im BGZ Wilhelminenspital wurden insgesamt 698 Patientinnen operiert, davon waren 42 Frauen mit familiärem Hochrisiko (6\%). 83 Brüste wurden rekonstruiert, davon 89\% (74 Brüste) risikoreduzierende Mastektomien, 9 Brüste wurden bei bereits bestehendem Karzinom operiert (11\%) Der Großteil der Rekonstruktionen fand mit Implantaten statt (65\%), davon 34\% (17) mit Expander- und 66\% (33) direct to implant (DTI) Rekonstruktionen. Im Vergleich wurden bei Patientinnen ohne Risiko mehrheitlich Eigengeweberekonstruktionen durchgeführt (59\%).

Die Komplikationsrate lag bei $14 \%$, wobei Brüste, die bereits an einem Karzinom erkrankt waren, eine signifikant höhere Anzahl komplikationsbedingter Folgeoperationen aufwiesen (33,3\% vs. 17,6\%). Ebenso hatten Brüste nach DTI Rekonstruktion signifikant häufiger Komplikationen als nach Expander-Rekonstruktionen (21\% vs. 11,7\%). Die Erfolgsrate lag bei Risikopatientinnen bei 96,4\%, 3 Brüste (3,6\%) mussten als Misserfolge verzeichnet werden.

Prophylaktische Mastektomien sollten ausschließlich bei Frauen mit familiärem Hochrisiko für Brustkrebsentstehung geführt werden. Diese Betroffenen müssen genauestens über das bestehende Risiko von Komplikationen aufgeklärt werden. Bei richtiger Indikationsstellung und Methodenwahl können onkologisch und ästhetisch nachhaltig gute Ergebnisse erzielt werden.

\section{4}

\section{Register von prophylaktischen Operationen bei Patientinnen} mit BRCA Mutation

Leser, C. ${ }^{1}$; Deutschmann, C. ${ }^{1}$; Dorffner, G. ${ }^{2}$; Abayev, S. ${ }^{3}$; Stübler, M. ${ }^{4}$; Reitsamer, . $^{5}$; Gschwantler-Kaulich, D. ${ }^{1}$; Singer, $C^{1}{ }^{1}$

${ }^{1}$ Abteilung für Gynäkologie und Geburtshilfe, Medizinische Universität Wien, Wien ${ }^{2}$ Zentrum für Medizinische Statistik, Informatik und Intelligente Systeme, Institut für Artificial Intelligence and Decision Support, Medizinische Universität Wien, Wien

${ }^{3}$ Plastische Chirurgie, Wilhelminenspital, Wien

${ }^{4}$ Abteilung für Chirurgie, Brustkompetenz Zentrum, KUK Linz, Linz

${ }^{5}$ Univ. Klinik für spezielle Gynäkologie, Paracelsus Medizinische Privatuniversität, Salzburg

Keywords: BRCA, Register, prophylaktische Mastektomie, prophylaktische Salpingo-Oophorektomie, Rekonstruktion

Hintergrund: Eine BRCA Mutation spricht für ein hohes Risiko an Brust- und/oder Eierstockkrebs zu erkranken. Der primäre Endpunkt des Registers ist die Erfassung der prophylaktischen Operationen bei Hochrisikopatientinnen in Österreich.

Sekundäre Endpunkte sind die Erfassung der verwendeten Techniken. Methodik: Die Datenerhebung seit 2015 erfolgte retro- und prospektiv. Es wurden Patientinnen aus dem AKH Wien, Hanusch Krankenhaus, Wilhelminenspital, LKH Salzburg und dem KUK Linz eingeschlossen.

Dieses Paper geht auf die ausschließlich prophylaktischen Operationen ein. Ein weiteres behandelt die Hochrisikopatientinnen mit aufgetretenen Karzinomen.

Ergebnisse: Insgesamt wurden 71 prophylaktisch operierte Patientinnen mit einer BRCA Mutation in das Register aufgenommen. Dabei wurde bei 41 (57\%) eine beidseitige prophylaktische Mastektomie (ME), bei 18 (25\%) eine prophylaktische bilaterale Salpingo-Oophorektomie (PBSO) und bei 13 (18\%) beides durchgeführt.

Eine Patientin erkrankte nach der prophylaktischen Operation an einem Karzinom.
Eine Rekonstruktion wurde bei 52 Patientinnen (96\% der Patientinnen mit ME) durchgeführt. 2 Patientinnen verzichteten auf eine Rekonstruktion. Alle wurden sofort rekonstruiert.

Die Implantateinlage erfolgte bei 35 Patientinnen subpectoral und bei 12 präpectoral. Wobei ein deutlicher Anstieg der präpectoralen Implantation in den letzten Jahren zu sehen ist. Das implantierte Volumen lag zwischen 155 und 650 CC (im Mittel 350 CC). Bei einer Erhöhung des Volumens um 100 CC verdoppelt sich die Wahrscheinlichkeit, dass Komplikationen auftreten. (Regressionskoeffizient -0,007).

8 Patientinnen wurden durch einen Expander rekonstruiert. 4 wurden durch Eigengewebe behandelt.

Zusammenfassung: Alle Patientinnen wurden sofort rekonstruiert. Wobei die meisten mittels Implantat wieder aufgebaut wurden.

Die Komplikationsrate verdoppelt sich mit der Erhöhung des Implantatvolumens um 100CC.

\section{5}

\section{Patient Satisfaction after Breast Cancer Surgery - a Prospective Clinical Trial}

\section{Leser, C. ${ }^{1}$ iTan, $Y^{1}{ }^{1}$; Zeillinger, R. $^{1}$; Fitzal, F. ${ }^{2}$; Singer, C. ' ; Gschwantler-} Kaulich, D.

${ }^{1}$ Abteilung für Gynäkologie und Geburtshilfe, Medizinische Universität Wien, Wien ${ }^{2}$ Klinische Abteilung für Allgemeinchirurgie, Universitätsklinik für Chirurgie, MedUni Wien, Wien

Keywords: Breast cancer, breast conserving surgery, oncoplastic breast surgery, breast reconstruction, quality of life, cosmetic result

Hintergrund: Die Brust ist ein Symbol für die Weiblichkeit. Ziel dieser Arbeit war es, herauszufinden, inwieweit die chirurgische Therapie des Mammakarzinoms die subjektiv empfundene Zufriedenheit mit dem kosmetischen Ergebnis betroffener Patientinnen beeinträchtigt und sich auf ihre Lebensqualität auswirkt.

Methodik: Einundsechzig Patientinnen (mittleres Alter 59 Jahre) wurden vor der Operation in diese prospektive Studie eingeschlossen. Die Datenerhebung, für die die Patientinnen die EORTC-QLQ-C-30-Fragebögen mit dem brustkrebsspezifischen Modul BR 23, einer zusätzlichen, an der Universität München konzipierten Frage, sowie den FBK R 23 von Herschbach ausfüllten, erfolgte an vier Zeitpunkten vor und nach dem Eingriff. $\mathrm{Zu}$ jedem dieser Zeitpunkte wurden zudem die Brüste der Patientinnen fotografiert und die Form und Proportion anhand der Fotos mit dem Breast Analyzing Tool (BAT) bestimmt. Zusätzlich wurden die Resultate von einem verantwortlichen Chirurgen sowie einem unabhängigen Brustspezialisten mittels Harris Scale bewertet.

Ergebnisse: Das 12-monatige Follow-up betrug 71\%; chirurgische Komplikationen waren in 8,2\% der Fälle zu verzeichnen. Die Brustasymmetrie war zum Zeitpunkt der Krankenhausentlassung am höchsten, die Lebensqualität (QoL) wurde am schlechtesten eingeschätzt. Beide Werte normalisierten sich im Laufe der folgenden 12 Monate. Chirurgen bewerteten das chirurgische Ergebnis signifikant besser als der unabhängige Brustspezialist $(\mathrm{p}=<0,001)$. Zudem waren ältere Patientinnen zum Zeitpunkt der Krankenhausentlassung signifikant weniger zufrieden mit dem kosmetischen Ergebnis ( $\mathrm{p}=0,024)$.

Zusammenfassung: Obwohl die Lebensqualität von Brustkrebspatientinnen nach der Diagnosestellung signifikant beeinträchtigt ist, ist dieser Effekt nicht von Dauer. Dies geht einher mit einer hohen Zufriedenheit mit dem kosmetischen Ergebnis, was in Bezug auf die Brustsymmetrie auch unabhängig verifiziert werden konnte. Die vorliegende Arbeit bestätigt damit den guten Standard der chirurgischen Therapie des Mammakarzinoms und liefert weitere Belege für die Annahme, dass die psychosoziale Belastung durch die Diagnose und Therapie mittelfristig abnimmt oder gar verschwindet. 
Danzinger, S. ; Kager, L. ${ }^{2}$; Bernathova, M. ${ }^{3}$; Lang, S. ; Haslik, W. ${ }^{5}$; Singer, $C .1$

${ }^{1}$ Universitätsklinik für Frauenheilkunde, Comprehensive Cancer Center, Medizinische Universität Wien, Wien

${ }^{2}$ Hämatologische und Onkologische Abteilung, St. Anna Kinderspital, Wien

${ }^{3}$ Univeristätsklinik für Radiologie und Nuklearmedizin, Comprehensive Cance

Center, Medizinische Universität Wien, Wien

${ }^{4}$ Klinisches Institut für Pathologie, Comprehensive Cancer Center, Medizinische Universität Wien, Wien

${ }^{5}$ Klinische Abteilung für Plastische und Rekonstruktive Chirurgie,

Universitätsklinik für Chirurgie, Comprehensive Cancer Center, Medizinische Universität Wien, Wien

Keywords: Osteoblastom, Mamma, Pädiatrie, Tumorresektion, Nachsorge Kategorie: Oral presentation/Poster

Topic: 5. Präinvasive Läsionen

Hintergrund: Wir berichten über ein 9-jähriges Mädchen mit einem Osteoblastom der Mamma. Da bislang über kein Osteoblastom der Mamma berichtet wurde, handelt es sich hierbei um eine Erstbeschreibung in der Weltliteratur.

Osteoblastom, ein gutartiger Knochentumor, ist selten mit einem Anteil von 14\% aller Knochentumore. Osteoblastome treten vorwiegend im Achsenskelett mit spinalen Läsionen auf. Sie sind zumeist auf den Knochen beschränkt, mit guter Prognose und niedriger Rezidivrate von ca. 15-20\%. Methoden: Fallbericht

Ergebnisse: Ein 9-jähriges Mädchen bemerkte seit einem Monat eine Schwellung in der linken Brust. In der primären Mammasonografie konnte eine Resistenz links laterokranial nachgewiesen werden. Die darauffolgende MR-Mammografie ergab BI-RADS 4 bei einer $27 \mathrm{~mm}$ messenden, teilweise glatt berandeten Herdläsion mit ausgeprägten Verkalkungen. In der ergänzenden Sonografie die Lymphknoten unauffällig. Die Ultraschallgezielte Biopsie ergab Material aus Knochenbälkchen, dies in erster Linie passend zu einem Osteoblastom des Weichgewebes. Das Staging mittels Ganzkörper-MRT sowie Abdomen-Sonografie war unauffällig.

Schließlich erfolgte die Tumorexstirpation mit unauffälligem postoperativem Verlauf und histologischer Bestätigung eines Osteoblastoms.

Zusammenfassung: Bei der erstmaligen Diagnose eines Osteoblastoms der Mamma erfolgte bei diesem Kind die chirurgische Resektion des Tumors mit ausgezeichnetem kosmetischem Ergebnis. Es sind Kontrolluntersuchungen alle 6 Monate mittels Ultraschalls geplant. Eine weitere systemische Therapie ist nicht indiziert.

07

\section{Accuracy of Surgical Opinion Case Report Forms to Predict Type of Axillary Surgery after Neoadjuvant Chemotherapy in the AGO-35 Trial}

\section{Sieghartsleitner, E. ${ }^{1}$; Peintinger, $F^{1,2}{ }^{2}$ Manurishi, N. ${ }^{2}$; Klaassen, $F^{3}{ }^{3}$;} Reitsamer, R. ${ }^{3}$; Kolberg, H.-C. ${ }^{4}$; Schmatloch, S. ${ }^{5}$; Kühn, T. ${ }^{6}$;AGO 35

${ }^{1}$ Pathologie, Institut für Pathologie, Medizinische Universität Graz, Graz

${ }^{2}$ Abteilung für Frauenheilkunde, LKH Leoben, Leoben

${ }^{3}$ Univ. Klinik für spezielle Gynäkologie, Paracelsus Medizinische

Privatuniversität, Salzburg

${ }^{4}$ Klinik für Gynäkologie und Geburtshilfe, Bottrop

${ }^{5}$ Brustzentrum am Elisabeth Krankenhaus Kassel, Kassel

${ }^{6}$ Klinik für Frauenheilkunde und Geburtshilfe, Esslingen

Keywords: Surgical opinion case report form, predicted axillary surgery, pathological complete response, prediction of response, axillary nodes Category: Poster

Topic: Anwendung prognostischer und prädiktiver Tests.

Purpose: To evaluate the accuracy of surgeon's opinion to predict axillary dissection (AND) or sentinel node biopsy (SLN) after neoadjuvant chemotherapy (NAC).

Methods: The prospective AGO-35 trial examines the validation of a genomic test to predict tumor response in the axilla. As part of the trial we used surgical opinion case report forms to predict the type of final axillary surgery based on clinicopathological and radiologic parameters in HER2 negative breast cancer patients. All patients received anthracycline- and taxane based neoadjuvant chemotherapy. Tumor stage was determined by the participating surgeons before NAC (t1) and prior to surgery (t2). We analyzed the agreement between predicted axillary surgery and pathological complete response (pCR).

Results: Of 205 patients, 161 complete surgical opinion case report forms were analyzed. Tumor stage $\geq \mathrm{T} 2$ at $\mathrm{t} 1$ was observed in $76 \%$ and nodal stage $\mathrm{N} \geq 1$ in $57 \%$. At final pathology $25 \%$ of the patients had a pCR in both the breast and axilla, 30\% in the breast. 101 patients $(63 \%)$ had a pCR in the axilla. Surgeons predicted a complete clinical response in the axillary nodes in 61,101 patients $(60 \%)$. SLN as final axillary surgery was performed in 60,101 (59\%). AND was performed in $41 \%$ of the patients who had a complete tumor response in the axilla.

Conclusion: Surgical opinion based on routine clinicopathological parameters showed a low accuracy to predict SLN. A genomic test, as evaluated in the AGO-35 trial, may predict tumor response in the axilla more accurately to spare patients with pCR from surgical overtreatment.

\section{Freier Vortrag}

\section{Expression des Androgenrezeptors in Mammakarzinom-Hirnmetastasen}

Bergen, E.S. ${ }^{1,2}$; Berghoff, A.S. ${ }^{1,2,3}$; Rajky, U. ${ }^{1,2}$; Rudas, M. ${ }^{1,4}$; Bago-Horvath, Z. ${ }^{1,4}$; Exner, R. ${ }^{1,5}$; Dieckman, K. ${ }^{1,6}$; Widhalm, G. ${ }^{1,7}$; Gnant, M. ${ }^{1,5}$; Zielinski, C.C. ${ }^{1}$; Steger, G.G. ${ }^{1,2}$; Preusser, M. ${ }^{1,2}$; Bartsch, R. ${ }^{1,2}$

${ }^{1}$ Comprehensive Cancer Center, Wien

${ }^{2}$ Klinische Abteilung für Onkologie, Universitätsklinik für Innere Medizin 1, Medizinische Universität Wien, Wien

${ }^{3}$ Universitätsklinik für Neuropathologie, Medizinische Universität Wien, Wien ${ }^{4}$ Universitätsklinik für Pathologie, Medizinische Universität Wien, Wien ${ }^{5}$ Universitätsklinik für Chirurgie, Medizinische Universität Wien, Wien ${ }^{6}$ Universitätsklinik für Strahlentherapie, Medizinische Universität Wien, Wien ${ }^{7}$ Universitätsklinik für Neurochirurgie, Medizinische Universität Wien, Wien

Keywords: Mammakarzinom, Hirnmetastasen, Androgenrezeptor, zielgerichtete Therapie, systemische Therapie

Hintergrund: Hirnmetastasen stellen eine spezielle Herausforderung in der Behandlung von Patientinnen mit Mammakarzinom dar und verkürzen das Gesamtüberleben aufgrund der limitierten Therapieoptionen. Der Androgenrezeptor (AR), welcher am häufigsten in luminalen Tumoren aber auch rund einem Drittel der triple-negativen Tumoren exprimiert wird, stellt ein potentielles Therapieziel dar. In der vorliegenden Studie haben wir somit die Expression des AR in Hirnmetastasen von Patientinnen mit Mammakarzinom und dessen Einfluss auf den klinischen Krankheitsverlauf analysiert.

Methoden: Patientinnen mit Mammakarzinom und neu diagnostizierten Hirnmetastasen wurden anhand des Vienna Brain Metastasis Registry identifiziert und deren klinische Daten einschließlich Patientencharakteristika, Subtyp und Gesamtüberleben retrospektiv erhoben. Formalin-fixierte und in Paraffin eingebettete Tumorblöcke von resezierten Hirnmetastasen der Neuro-Biobank wurden verwendet, um die immunhistochemische Färbung des AR durchzuführen und anschließend die Expression zu analysieren. Ergebnisse: Insgesamt standen 46 Hirnmetastasen-Proben zur Analyse zur Verfügung. In 15 von 46 (32,6\%) der Proben konnte eine AR-Expression $\geq 1 \%$ nachgewiesen werden. Die mediane Expressionsrate betrug $1 \%$ (Schwankungsbreite 0-60). Eine nicht-signifikant höhere AR-Expression konnte in 9 von 18 (50,0\%) der luminalen, in 4 von 16 (25,0\%) der Her2positiven und in 2 von $12(16,7 \%)$ der triple-negativen Hirnmetastasen nachgewiesen werden $(\mathrm{p}>0,05)$. Eine Korrelation zwischen dem medianen Hirnmetastasen-freien Gesamtüberleben und der AR-Expression lag nicht vor (HR 1,0; 95\%-KI 1,0-1,0; p > 0,05). Das mediane Gesamtüberleben ab Diagnose der Hirnmetastasen betrug 9 Monate (Schwankungsbreite 0-104). Eine Assoziation zwischen dem medianen Gesamtüberleben und der AR-Expression konnte nicht festgestellt werden ( 9 vs. 10 Monate; HR 1,0; 95\%-KI 1,0-1,0; p > 0,05; log rank test). 
Zusammenfassung: Entsprechend unserer Analyse, wird der AR in einem Drittel der Mammakarzinom-Hirnmetastasen mit der höchsten Inzidenz im luminalen Subtyp exprimiert. Somit könnte der AR auch in der Behandlung dieser speziellen Patientenpopulation eine mögliche Therapiegrundlage bieten.

\section{Freier Vortrag}

Die Auswirkungen der Resektionsränder nach neoadjuvanter Chemotherapie auf das Outcome bei Mammakarzinomen eine retrospektive Studie

Bolliger, $M .{ }^{1}$; Wimmer, $K^{1}{ }^{1}$; Exner, $R .{ }^{1}$; Helfgott, $R{ }^{2}{ }^{2}$; Heck, $D .{ }^{2}$; Schlagnitweit, $P^{2}{ }^{2}$; Mittlböck, M. ${ }^{3}$; Függer, R. ${ }^{2}$; Gnant, M. ${ }^{1}$; Fitzal, F. ${ }^{1}$

${ }^{1}$ Klinische Abteilung für Allgemeinchirurgie, Universitätsklinik für Chirurgie, MedUni Wien, Wien

${ }^{2}$ Abteilung für Allgemein- und Viszeralchirurgie, Ordensklinikum Linz, Linz ${ }^{3}$ Informatik und Intelligente Systeme, Zentrum für Medizinische Statistik, MedUni Wien, Wien

Keywords: Mammakarzinom, Neoadjuvant, Resektionsrand, Lokalrezidiv, Pathologisch komplette Remission

Kategorie: Oral presentation oder poster

Topics: Loko-regionäre Therapie

Hintergrund: Bei primär operierten Patientinnen gilt «no tumor on ink» als freier Resektionsrand. Für die Definition eines freien Resektionsrandes nach neoadjuvanter Chemotherapie gibt es deutlich weniger Daten. Wir verglichen das Lokalrezidiv zwischen knappem Resektionsrand $(<1 \mathrm{~mm})$, freiem Resektionsrand ( $>1 \mathrm{~mm}$ ) mit pathologisch kompletter Resektion, bei der gar keine Resektionsrandangabe getroffen werden kann.

Methoden: Wir haben retrospektiv Daten von Patientinnen erfasst, welche zwischen 1994 und 2014 an der Medizinischen Universität Wien und dem Krankenhaus der Barmherzigen Schwestern in Linz operiert wurden. Eingeschlossen wurden nur brusterhaltend operierte Patientinnen nach neoadjuvanter Chemotherapie und R0 Resektion.

Ergebnisse: Von den 406 auswertbaren Patientinnen hatten 282 eine R0 Resektion $>=1 \mathrm{~mm}, 74<1 \mathrm{~mm}$ und 50 hatten eine RX-Resektion (pathologisch komplette Remission). R0 gegenüber RX hatten keinen signifikanten Unterschied im Overall-Surival ( $\mathrm{p}=0,1457$, Trend $\mathrm{zu} \mathrm{Rx}$ ), ebenso im lokalrezidivfreien Überleben $(p=0,633)$. Das disease-free survival war mit $\mathrm{p}=0,0583$ zugunsten von Rx einer Signifikanz am Nächsten.

Zusammenfassung: In dieser kleinen hypothesengenerierenden Analyse zeigte sich kein Unterschied im onkologischen Ergebnis zwischen verschiedenen R0 Definitionen. Weitere Analysen mit größeren Fallzahlen sind nötig, um mehr über die klare Definition eines negativen Resektionsrandes nach neoadjuvanter Chemotherapie aussagen zu können.

10

Prophylaktische Mastektomien und bilaterale SalpingoOophorektomien bei Patientinnen mit Mammakarzinom eine Registerstudie

Deutschmann, C. ${ }^{1}$; Leser, C. '; Dorffner, G. ${ }^{2}$; Gschwantler-Kaulich, D. ${ }^{1}$; Abayev, S. ${ }^{3}$; Reitsamer, R. ${ }^{4}$; Stübler, M. ${ }^{5}$; Singer, C.F. ${ }^{1}$

${ }^{1}$ Klinische Abteilung für Allgemeine Gynäkologie und Gynäkologische Onkologie, Universitätsklinik für Frauenheilkunde, MUW, Wien

${ }^{2}$ Institut für Artificial Intelligence and Decision Support, MUW, Wien

${ }^{3}$ Abteilung für Plastische, Ästhetische und Rekonstruktive Chirurgie, Wilhelminenspital, Wien

${ }^{4}$ Universitätsklinik für Frauenheilkunde und Geburtshilfe, PMU, Salzburg

${ }^{5}$ Abteilung für Chirurgie, Brustkompetenz Zentrum, KUK Linz, Linz

Keywords: Prophylaktische Mastektomie, PBSO, BRCA, Mammakarzinom, Registerstudie

Kategorie: Oral presentation/poster

Topics: Prophylaktische Mastektomie

Hintergrund: Die vorliegende Registerstudie soll die Anwendung prophylaktischer Mastektomien (ME) und bilateraler Salpingo-Oophorekto- mien (PBSO) bei BRCA-positiven Patientinnen mit einem Mammakarzinom in der Anamnese in Österreich aufzeigen.

Methoden: Patientinnen wurden an 4 Studienzentren (AKH Wien, WSP, SALK und KUK Linz) rekrutiert.

Ergebnisse: Insgesamt wurden 111 Patientinnen eingeschlossen. 86,8\% der Patientinnen, die eine kurativ unilaterale $\mathrm{ME}$ erhielten, ließen zusätzlich eine prophylaktische ME der kontralateralen Seite durchführen. Patientinnen, die eine MRM und/oder eine Sofortrekonstruktion erhielten, ließen statistisch signifikant häufiger eine prophylaktische ME kontralateral durchführen $(\mathrm{p}=0,001) .52 \%$ der Patientinnen erhielten eine PBSO. Patientinnen mit PBSO hatten statistisch signifikant seltener eine prophylaktische ME in der Anamnese ( $\mathrm{p}=0,001)$.

Zusammenfassung: Der hohe Anteil an kurativ unilateralen ME in Kombination mit prophylaktisch unilateralen ME könnte auf eine effiziente Aufklärung über die Risiko-reduzierende Wirkung einer prophylaktischen ME auf das Auftreten eines Mammakarzinoms bei Vorliegen einer BRCA-Mutation hinweisen.

Eine größere Tumorausdehnung, die eine MRM nach sich zieht, beziehungsweise die Aussicht auf die Wiederherstellung der natürlichen Brustform ist eventuell mit einer erhöhten Bereitschaft der Patientinnen zur prophylaktischen Operation assoziiert.

Der hohe Anteil an Patientinnen mit PBSO ohne prophylaktische ME in der Anamnese ist eventuell auf die bereits Risiko-reduzierende Wirkung einer PBSO auf das Auftreten eines Mammakarzinoms zurückzuführen.

\section{Freier Vortrag}

\section{Präpektorale versus Retropektorale Implantatrekonstruktion - ein Vergleich der Methoden-assoziierten Komplikationsraten}

\section{Deutschmann, C. ; Dorffner, G. ${ }^{2}$; Singer, C.F. ${ }^{1}$; Leser, C. ${ }^{1}$; Gschwantler-} Kaulich, D.

${ }^{1}$ Klinische Abteilung für Allgemeine Gynäkologie und gynäkologische Onkologie, Universitätsklinik für Frauenheilkunde, Medizinische Universität Wien, Wien

${ }^{2}$ Informatik und Intelligente Systeme, Zentrum für Medizinische Statistik Institut für Artificial Intelligence and Decision Support, Medizinische Universität Wien, Wien

Keywords: Präpektorale Implantatrekonstruktion, Retropektorale Implantatrekonstruktion, Komplikationen, Komplettierungsoperationen, angleichende Operationen

Kategorie: Oral presentation/poster

Topics: Update Mammachirurgie

Hintergrund: Die Weiterentwicklung operativer Techniken auf dem Gebiet der Mastektomie sowie die Einführung dermaler Matrices und synthetischer Netze haben zu der Wiederentdeckung der präpektoralen Implantatrekonstruktion geführt. Komplikationen der etablierten retropektoralen Implantatpositionierung als Folge der Durchtrennung des Musculus pectoralis sollen so potentiell vermieden werden.

Methoden: Das primäre Studienziel war der retrospektive Vergleich der Komplikationsraten eine Woche, ein Monat und ein Jahr nach präpektoraler versus retropektoraler Implantatrekonstruktion bei Patientinnen, die zwischen 2013 und 2017 eine Implantat-basierte Brustrekonstruktion an der Universitätsklinik für Frauenheilkunde des AKH Wien erhalten hatten. Ergebnisse: Insgesamt wurden 57 Patientinnen (94 Brüste) präpektoral und 95 Patientinnen (149 Brüste) retropektoral rekonstruiert. Die 2 Kohorten zeigten keinen statistisch signifikanten Unterschied in der Rate an Komplikationen (Dehiszenz, Infektion, Serom, Nachblutung, Nekrose, Fistel, Kapselkontraktur und Rippling), Reinterventionen (Serompunktion und Sekundärnaht), Reoperationen (Nachblutung und Nekrosektomie) beziehungsweise Implantatverlusten. Es gab keinen statistisch signifikanten Unterschied in der Anzahl an ergänzend durchgeführten Komplettierungsoperationen ipsilateral (Narbenkorrektur und Lipofilling) beziehungsweise bei einseitig erfolgter Implantatrekonstruktion angleichenden Operationen kontralateral (Mastopexie, Augmentation und Lipofilling). Zusammenfassung: Die präpektorale Implantatrekonstruktion erlaubt die Rekonstruktion der Brust mit gegenüber der konventionellen submuskulären Implantatpositionierung vergleichbaren Komplikationsraten, 
während - wie in früheren Studien gezeigt - die auf der Loslösung des Muskels beruhenden Nachteile der retropektoralen Implantatrekonstruktion vermieden werden können.

12

Der singuläre Rundherd beim Mammacarzinom! Immer eine Metastase? Herausforderung an die interdisziplinäre Zusammenarbeit zwischen Senologie und Thoraxchirurgie

Themel, M.; Gabor, S.E.; Niernberger, T.; Sauseng, S.; Strohmeyer, K.; Herz, W.; Rabl, $\mathrm{H}$.

Abteilung für Chirurgie, LKH Leoben, Leoben

Keywords: Mammakarzinom, Lungenrundherd, pulmonale Metastasen, Differentialdiagnose

Hintergrund: Der pulmonale Rundherd bei Patientinnen mit Mammacarzinom zählt zu den häufigsten Begleitdiagnosen sowohl bei der Erstmanifestation als auch in der onkologischen Nachsorge. Die einfachste Konsequenz wäre diesen Rundherd in Zusammenhang mit der Tumorerkrankung der Brust zu sehen. Die Erfahrungen der letzten Jahre zeigen uns aber, dass das Naheliegendste nicht immer das Richtige ist.

Methoden/Ergebnisse: Aufarbeitung von 4 Fällen:

Im ersten Fall erfolgte simultan die Diagnostik eines Mammacarzinoms und eines singulären Rundherdes der Lunge. Es wurde primär eine CT gezielte Punktion des Herdes durchgeführt. Das Ergebnis zeigte einen Primärtumor der Lunge und die Patientin wurde sowohl einer brusterhaltenden OP als auch einer Lobektomie der Lunge zugeführt. Im zweiten Fall zeigt sich ein größenstationärer glatt begrenzter Lungenrundherd in der onkologischen Nachsorge. Es wurde eine Keilexcision der Lunge durchgeführt - die Histologie ergab ein Karzinoid der Lunge. Im dritten Fall präsentiert sich in der Nachsorge ein unregelmäßig begrenzter Herd im apikalen Oberlappen der rechten Lunge, der in der PET signifikant speicherte. Die Patientin wurde operativ saniert und das Ergebnis war ein Tuberkulom. Im vierten Fall zeigt sich in der Nachsorge ein spekulierter unregelmäßig begrenzter Herd mit hilär vergrößerten Lymphknoten. Die Patientin wurde operiert und das Ergebnis war eine MammakarzinomMetastase.

Zusammenfassung: Die histologische Diagnosesicherung eines Lungenrundherdes sollte Standard sein. Eine Besprechung dieser Fälle mit dem Thoraxchirurgen/in stellt eine wichtige Säule in der Therapie dar.

13

\section{Granularzelltumor der Brust - eine Rarität}

Strohmeyer, K. ${ }^{1}$; Herz, W. ${ }^{1}$; Themel, M. ${ }^{1}$; Uggowitzer, M. ${ }^{2}$; Tötsch, M. ${ }^{3}$; Rabl, . $^{1}$

${ }^{1}$ Abteilung für Chirurgie, LKH Hochsteiermark, Standort Leoben, Leoben

${ }^{2}$ Abteilung für Radiologie, LKH Hochsteiermark, Standort Leoben, Leoben

${ }^{3}$ Abteilung für Pathologie, LKH Hochsteiermark, Standort Leoben, Leoben

Keywords: Seltene Entität, benigne, imponieren malignitätsverdächtig, Exzision, kein SNB

Vortragsform: Poster

Hintergrund: Granularzelltumore sind benigne Tumore, die von perineuralen Zellen abstammen. Am häufigsten treten diese Entitäten im Hals-/ Nackenbereich auf und nur sehr selten in der Brustdrüse. Meist lokalisiert im oberen, inneren Quadranten sowie sternumnahe. Betroffen sind gewöhnlicherweise prämenopausale Frauen. Sowohl in der klinischen Untersuchung als auch in der Bildgebung imponieren diese Tumore malignitätsverdächtig, insbesondere aufgrund der schlechten Abgrenzbarkeit. Methoden und Ergebnisse: Wir berichten über eine junge Patientin, der ein größenprogredienter, schlecht verschieblicher Knoten im oberen, inneren Quadranten der rechten Mamma auffiel.

Die weitere bildgebende Abklärung zeigte einen ca. $1 \mathrm{~cm}$ großen Knoten, der als BI-RADS IV klassifiziert wurde. Aufgrund dessen wurde die Indikation zur ultraschallgestützten Stanzbiopsie gestellt. Die mikroskopische und immunhistochemische Aufarbeitung (S-100 Expression positiv) ergab einen Granularzelltumor der Brustdrüse.

Als Therapie der Wahl gilt die Exzision des Tumors. Eine axilläre Lymphadenektomie und eine weitere adjuvante Chemo- oder Strahlentherapie sind nicht erforderlich.

Zusammenfassung: Granularzelltumore der Mamma sind seltene, benigne Tumore, die sowohl in der klinischen Untersuchung als auch in der Bildgebung malignitätsverdächtig erscheinen. Die Therapie der Wahl ist eine vollständige Exzision mit Sicherheitsabstand, aufgrund der geringen Wahrscheinlichkeit einer Entartung.

\section{4}

\section{Einfluss soziodemografischer Faktoren auf eine Brustrekonstruktion nach Mastektomie. Eine prospektive Beobachtungsstudie}

\section{Stolz, M. ${ }^{1}$; Farr, A. ${ }^{1}$; Deutschmann, C. ${ }^{1}$; Baumann, L. ${ }^{2}$; Singer, C.F. ${ }^{1}$, Tea,} M.-K.M. ${ }^{1}$

${ }^{1}$ Brustgesundheitszentrum, Universitätsklinik für Frauenheilkunde Medizinische Universität Wien, Wien

${ }^{2}$ Informatik und intelligente Systeme, Zentrum für medizinische Statistik,

Medizinische Universität Wien, Wien

Fragestellung: Trotz optimierter Verfahren gibt es eine beträchtliche Anzahl von Patientinnen, die eine Brustrekonstruktion nach Mastektomie ablehnen. In dieser Arbeit wird über Faktoren berichtet, die die Entscheidung für eine Rekonstruktion nach Mastektomie beeinflussen.

Methodik: Mittels Fragebogen erfolgte eine prospektive Erhebung soziodemografischer Daten aller Frauen, bei denen von 2016-2017 an der Universitätsklinik für Frauenheilkunde (Wien) eine Mastektomie durchgeführt wurde. Der Einfluss einzelner Co-Variablen auf die Rekonstruktion wurde mit logistischer Regression berechnet.

Ergebnisse: Insgesamt erhielten 137 Patientinnen eine Mastektomie, von denen 120 mit 131 Mastektomien in die Studie inkludiert wurden. Auf 77 (58,8\%) der Mastektomien folgte eine Rekonstruktion, auf 54 (41,2\%) keine. Implantate waren die häufigsten Rekonstruktionen. Das durchschnittliche Alter lag bei 53,1 Jahren. Jene mit bzw. ohne Rekonstruktion waren im Mittel 46,9 bzw. 60 Jahre alt (OR =0,93; CI = 0,899-0,936; $\mathrm{p}=<0,001) ; 81(78,6 \%)$. Die häufigste höchste abgeschlossene Schulbildung war die Universität/FH ( $\mathrm{p}=0,44)$. Es lebten 76 (74,5\%) Pat. zur Zeit der Mastektomie in einer Partnerschaft, davon 42 (56\%) mit Rekonstruktion, 33 (44\%) ohne ( $\mathrm{p}=0,13)$. Bezüglich dem Monatseinkommen zeigte sich ebenso kein signifikanter Unterschied $(p=0,41)$.

Schlussfolgerung: Je jünger die Patientin, desto höher ist die Wahrscheinlichkeit, dass sie sich für eine Rekonstruktion entscheidet. Neben dem Alter scheinen soziodemografische Faktoren keinen Einfluss auf die Wiederaufbaurate zu haben.

\section{Freier Vortrag}

\section{Nipple sparing Mastektomie für alle?}

Retrospektive Daten des Brustzentrum Salzburg mit 5 Jahren Follow-up in Bezug auf kosmetisches Outcome, Patientinnenzufriedenheit, Komplikationen und Rezidivraten

Alshaibani, N.; Klaassen Federspiel, F.; Sir, A.; Gschwandtner, E.; Reitsamer, R. Frauenheilkunde und Gynäkologie, Brustzentrum - spez.Gyn., Universitätsklinik der PMU Salzburg, Salzburg

Keywords: Nipplesparing Mastektomie, Lokalrezidive, onkologische Sicherheit, Komplikationsraten, kosmetisches Outcome

In den letzten Jahren haben die SSME und NSME die konventionelle Mastektomie bei korrekter Indikationsstellung weitestgehend abgelöst. Die SSME ist der konventionellen Mastektomie in Bezug auf lokale Rezidivraten gleichwertig. Bei NSME wurden in der Literatur höhere Rezidivraten beschrieben, was einerseits auf den Erhalt des NAC zurückgeführt 
wurde. Stellt der Erhalt des Nippel Areola Komplexes eine vermeidbare Gefahr für die Patientin dar?

Es wurden retrospektive Daten aller Patientinnen nach NSME in einem Zeitraum von März 2011 bis November 2016 analysiert. Bei 140 Patientinnen wurden 200 NSME durchgeführt.

Nach einem medianen Follow-up von 26 Monaten zeigte sich bei 8138 Patientinnen ein Lokalrezidiv, keines davon im erhaltenen NAC, daraus ergibt sich eine Lokalrezidivrate von 5,7\%. Im Follow-up-Zeitraum wurden bei 8 weiteren Patientinnen Fernmetastasen diagnostiziert. Bei diesen Patientinnen handelte es sich vorwiegend um jüngere Frauen und $\mathrm{Tu}-$ morerkrankungen mit ungünstiger Tumorbiologie.

Mit zunehmenden Fallzahlen konnte in diversen Studien gezeigt werden, dass der Erhalt des NAC nach unauffälligem nipple coring keine relevante Gefahr in Bezug auf das Lokalrezidivrisiko darstellt. Unsere Daten decken sich diesbezüglich mit den Ergebnissen internationaler Studien.

Ob eine Gruppe von Patientinnen mit hohem Rezidivrisiko dennoch von einer konventionellen Mastektomie profitiert, muss in größer angelegten Studien verifiziert werden.

\section{Freier Vortrag}

\section{AGO R02 Brustrekonstruktion nach Mastektomie - Register} zur prospektiven und retrospektiven Erfassung und Dokumentation von Brustrekonstruktionen nach NippleSparing-Mastektomie

Klaassen-Federspiel, F. ${ }^{1}$; Pristauz, G. ${ }^{2}$; Sieghartsleitner, E. ${ }^{3}$; Koller, R. ${ }^{4}$; Sir, A. ${ }^{1}$; Reitsamer, $R .{ }^{1}$; Peintinger, $F^{3,5}$

${ }^{1}$ Frauenheilkunde und Gynäkologie, Brustzentrum, Universitätsklinik der PMU Salzburg, Salzburg

${ }^{2}$ Klinische Abteilungen für Gynäkologie, LKH-Univ.-Klinikum, Medizinische Universität Graz

${ }^{3}$ Pathologie, Med. Univ. Graz, Institut für Pathologie, Graz

${ }^{4}$ Plastische Chirurgie, Wilhelminenspital, Wien

${ }^{5}$ Abteilung für Frauenheilkunde, LKH Leoben, Leoben

Konservative Mastektomietechniken wie Nipple-Sparing-Mastektomie (NSM) und Skin-Sparing-Mastektomie (SSM) ersetzen zunehmend die konventionelle Mastektomie. Daten zu Frequenz, Indikation, Operationstechnik, Komplikationen und Ergebnissen liegen für Österreich nicht vor Ein Register zur prospektiven und retrospektiven Erfassung und Dokumentation von Brustrekonstruktionen nach Nipple-Sparing-Mastektomie wurde implementiert.

Folgende Daten werden erfasst: Patientencharakteristika, Voroperationen, Vortherapien, Operationsindikation, Operationsart und Technik, Rekonstruktionsart, Implantatmodell, Implantatgröße, Implantatlage, Brustvolumen und Gewicht, Liegedauer Drainage, Antibiotikatherapie, eingesetzte Matrix, histologisches Ergebnis, sowie postoperative Therapie, Komplikationen, Revisionsoperationen, kosmetisches Ergebnis, followup, lokales und lokoregionäres Rezidiv, sowie Fernmetastasierung. Bisher wurden Daten von 65 Patientinnen und 127 Mastektomien (62 Patientinnen bilateral) erfasst:

\begin{tabular}{|lcc|}
\hline & $\mathrm{n}$ & $\%$ \\
\hline Operationsart & & \\
\hline NSM & 97 & $77,6 \%$ \\
\hline SSM & 26 & $20,8 \%$ \\
\hline andere & 2 & $1,6 \%$ \\
\hline Rekonstruktion & & \\
\hline Implantat & 124 & $97,6 \%$ \\
\hline Autolog & 3 & $2,3 \%$ \\
\hline Matrix & 112 & $90,3 \%$ \\
\hline Strattice & 9 & $8,0 \%$ \\
\hline Surgimend & 46 & $41,1 \%$ \\
\hline Tigr & 54 & $48,2 \%$ \\
\hline Tiloop & 2 & $1,8 \%$ \\
\hline andere & 1 & $0,8 \%$ \\
\hline
\end{tabular}

\begin{tabular}{|lcc|}
\hline & $\mathrm{n}$ & $\%$ \\
\hline Position & & \\
\hline präpektoral & 4 & $3,3 \%$ \\
\hline subpektoral & 118 & $96,7 \%$ \\
\hline Komplikationen & & \\
\hline Total & 16 & $13,1 \%$ \\
\hline Revisionen & 11 & $9,0 \%$ \\
\hline Implantatverlust & 1 & $0,8 \%$ \\
\hline Kosmesis & & \\
\hline sehr gut und gut & 51 & $79,7 \%$ \\
\hline zufriedenstellend & 9 & $14,1 \%$ \\
\hline schlecht & 4 & $6,2 \%$ \\
\hline
\end{tabular}

Im Sinne einer umfassenden Erfassung der österreichischen Ergebnisse werden alle Kliniken, die Brustrekonstruktionen durchführen, aufgerufen, an diesem Register teilzunehmen.

18

\section{Neoadjuvante Chemotherapie bei Patientinnen mit Mammakarzinom - eine retrospektive Studie}

Suppan, C. ${ }^{1,2}$; Matzenauer, E. ${ }^{1,2}$; Brcic, I. ${ }^{3}$; Mueller, D.H. ${ }^{1,2}$; Dandachi, N. ${ }^{1,2}$; ${\text { Balic, } M{ }^{1,2}}^{1, i}$

${ }^{1}$ Klinische Abteilung für Onkologie, Universitätsklinik für Innere Medizin, LKHUniv. Klinikum Graz, Graz

${ }^{2}$ Comprehensive Cancer Center Graz, Graz

${ }^{3}$ Institut für Pathologie, Medizinische Universität Graz, Graz

Hintergrund: Brustkrebs ist österreichweit die häufigste Krebserkrankung der Frau. Neben der chirurgischen Therapie steht vor allem die Chemotherapie im Fokus der Behandlung. Ziel dieser Arbeit war es, herauszufinden, welche neoadjuvanten Chemotherapien verabreicht worden sind und in welchem Ausmaß es zu Dosisreduktionen gekommen ist.

Methode: Insgesamt wurden 213 Patientinnen, die eine neoadjuvante Therapie im Zeitraum von 2011 bis 2016 erhalten haben, retrospektiv untersucht. Ein besonderes Augenmerk wurde auf die geplante und tatsächlich verabreichte neoadjuvante Chemotherapie gelegt.

Ergebnisse: Es konnte bei dieser Studie ein eindeutiger Zusammenhang zwischen dem Alter und der Dosierung der neoadjuvanten Chemotherapie hergestellt werden. So wurden hauptsächlich ältere Patientinnen von vornherein mit einer niedrigeren Dosierung behandelt. Beim wöchentlichen Taxol kam es häufig zum vorzeitigen Abbruch der geplanten Therapie.

Schlussfolgerung: $\mathrm{Ob}$ und welchen Einfluss eine solche Dosisreduktion auf das Gesamtüberleben haben könnte, lässt sich anhand dieser Arbeit nicht erheben. Trotzdem sollte das Hauptziel der Chemotherapie von Brustkrebspatientinnen eine möglichst optimale Dosierung sein, die sicher und mit möglichst wenigen Komplikationen verabreicht werden kann. 
Langzeitergebnisse von Patientinnen mit einem primären Mammakarzinom - eine retrospektive Studie

Suppan, C. ${ }^{1,2}$; Pesendorfer, N. ${ }^{1,2}$; Mueller, D.H. ${ }^{1,2}$; Hammer, R. ${ }^{3}$; Prein, $K^{4}$; Hauser, H. ${ }^{3}$; Dandachi, N. ${ }^{1,2}$; Balic, M. ${ }^{1,2}$

${ }^{1}$ Klinische Abteilung für Onkologie, Universitätsklinik für Innere Medizin, LKHUniv. Klinikum Graz, Graz

${ }^{2}$ Comprehensive Cancer Center Graz, Graz

${ }^{3}$ Department Chirurgie, Landeskrankenhaus Graz Süd-West, Graz

${ }^{4}$ Institut für Pathologie, Landeskrankenhaus Graz Süd-West, Graz

Mit 29\% aller Malignome ist das Mammakarzinom der häufigste maligne Tumor der Frau. Für die Therapie des Mammakarzinoms stehen mehrere, durch Leitlinien vorgegebene Behandlungsmethoden zur Verfügung, die je nach Patient an dessen Bedürfnisse angepasst werden.

Ziel dieser Arbeit war es, die Zusammensetzung der primären Mammakarzinom-Fälle, die im Zeitraum von 2011 bis 2016 in Brustgesundheitszentrum Graz-West diagnostiziert wurden und auf der Klinischen Abteilung für Onkologie behandelt wurden, hinsichtlich der bekannten biologischen Untergruppen darzustellen und aufzuzeigen, wie sich jene Brustkrebsarten nach Behandlungsabschluss verhalten. Folgende für das Mammakarzinom definierten Outcome-Parameter wurden herangezogen: Gesamtüberleben (OS), brustkrebsspezifisches Überleben (BCSS), krankheitsfreies Überleben (DFS) und das Fernmetastasen-freie Überleben (DDFS).

Insgesamt wurden 801 Patientinnen in diese Studie miteinbezogen. Die primäre Therapiestrategie war bei 192 Patientinnen neoadjuvant, bei 539 adjuvant und bei 70 palliativ. Die mediane Nachbeobachtungszeit war 3,8 Jahre und in diesem Zeitraum sind insgesamt 135 Patientinnen (unabhängig von der Todesursache) verstorben. Das OS für alle Patientinnen betrug nach 3 Jahren 85\%, für die adjuvante Population 93\%, die neoadjuvanten Patienten $85 \%$ und für die palliativen Patienten 21\%. Das DFS der adjuvanten Patientinnen betrug nach 3 Jahren 93\% und das DDFS 97\%. Das erreichte DFS/DDFS und OS unserer Patientinnen ist erwartungsgemäß und lässt auf eine gute Versorgungsqualität rückschließen.

20

\section{Häufigkeit von BRCA1,2-Mutationen in einer retrospektiv untersuchten Kohorte in der Hochsteiermark}

Simon, K. ${ }^{1}$; Peintinger, F. ${ }^{1,2}$; Geigl, J.B. ${ }^{3}$; Singer, C. ${ }^{4}$; Tamegger, A. ${ }^{1}$; Knöbelreiter, S. ${ }^{1}$

${ }^{1}$ Abteilung für Gynäkologie und Geburtshilfe, LKH Hochsteiermark, Standort Leoben, Leoben

${ }^{2}$ Institut für Pathologie, Medizinische Universität Graz, Graz

${ }^{3}$ Institut für Humangenetik, Medizinische Universität Graz, Graz

${ }^{4}$ Zentrum für Familiären Brust- und Eierstockkrebs, AKH Wien, Wien

Keywords: Hereditäres Mammakarzinom, BRCA1,2-Mutation, VUS, Tumorsuppressorgene, Genetische Testung.

Kategorie: Poster.

Topics: 7. Genetische Prädisposition, Tools.

Hintergrund: 5-10\% aller Mammakarzinome basieren auf einem genetischen Hintergrund. Knapp die Hälfte dieser hereditären Karzinome wird durch Mutationen in den Tumorsuppressorgenen BRCA1 und BRCA2 hervorgerufen. Etwa 10-20\% dieser BRCA1,2-Veränderungen werden als Varianten unbestimmter Signifikanz (VUS) geführt, deren Pathogenität mit ihrem möglich assoziierten Erkrankungsrisiko aktuell noch nicht sicher zugeordnet werden kann.

Methoden: In einer retrospektiven Analyse wurden die Daten von 180 genetisch getesteten Personen im Brustgesundheitszentrum an der gynäkologischen Abteilung des LKH Hochsteiermark - Standort Leoben evaluiert. Die genetischen Testungen wurden am Institut für Humangenetik der Medizinischen Universität Graz und im Zentrum für Familiären Brust- und Eierstockkrebs des AKH Wien zwischen 01/2010 und 04/2017 durchgeführt. Bei einer bereits bekannten familiären Mutation erfolgte eine Einzelgen-Diagnostik oder einzelne ausgewählte Gene wurden se- quenziert oder eine Testung im Rahmen des Next Generation Sequencing (NGS) Tumorpanel wurde durchgeführt. Die Häufigkeit von BRCA1,2Mutationen und VUS wurden objektiviert und deren Assoziation zum Auftreten von Mammakarzinomen unter Berücksichtigung von Tumorstadium, Erkrankungsalter und Histopathologie ermittelt.

Ergebnisse: 22 BRCA1 (16\%)- und 14 BRCA2-Mutationsträgerinnen (10\%) identifiziert. Insgesamt konnten 22 VUS festgestellt werden. Im untersuchten Gesamtkollektiv $(\mathrm{n}=135)$ waren zum Zeitpunkt der genetischen Testung 88 Personen an einem Mammakarzinom erkrankt (67 unilateral, 14 bilateral, 6 Mamma- Ovarialkarzinom). 11 von 88 erkrankten Personen wurden positiv auf eine BRCA1- und 11 wurden positiv auf eine BRCA2-Mutation getestet. Mutationsträgerinnen erkrankten durchschnittlich mit 43 (BRCA1) bzw. 47 Jahren (BRCA2) und diese mit nachgewiesener VUS im Alter von 48 Jahren an einem Mammakarzinom. 73\% der BRCA1-assoziierten Mammakarzinome waren schlecht differenziert (G3) und hatten einen hohen Proliferationsindex (Ki-67-Index median 48\%). Mehr als die Hälfte der Mammakarzinome waren Hormon- und Her2/neu-Rezeptor negativ. 54\% der BRCA2-assoziierten Mammakarzinome waren schlecht differenziert und 63\% waren hormonrezeptorpositiv. VUS-assoziierte Mammakarzinome waren histopathologisch am ähnlichsten zu den sporadisch aufgetretenen Mammakarzinomen.

Zusammenfassung: Das hereditäre Mammakarzinom stellt eine komplexe Erkrankung dar, die sich in Ätiologie, Diagnostik, Histopathogenese, Prävention und auch in der Therapie von sporadischen Karzinomen unterscheidet. Der Stellenwert der VUS stellt aufgrund des unklaren Risikoprofils eine große Herausforderung in der genetischen Beratung.

\section{1 \\ Sentinel Node Markierung bei Mammakarzinom: Was bringt das Spätbild?}

Taumberger, N. ${ }^{1}$; Pernthaler, B. ${ }^{2}$; Schwarz, T. ${ }^{2}$; Bjelic-Radisic, V. ${ }^{1}$; Pristauz, G. ${ }^{1}$; Aigner, R.M. ${ }^{2}$; Tamussino, K. ${ }^{1}$

${ }^{1}$ Klinische Abteilungen für Gynäkologie, LKH-Univ. Klinikum, Medizinische Universität Graz, Graz

${ }^{2}$ Klinische Abteilungen für Nuklearmedizin, LKH-Univ. Klinikum, Medizinische Universität Graz, Graz

Fragestellung: Die Sentinel Node Biopsie (SNB) ist heute Standard bei der operativen Therapie bzw. beim axillären Staging des Mammakarzinoms. Nichtsdestotrotz sind internationale Leitlinien zum genaueren Procedere der SNB in Bezug auf Technik, z.B. Spätbild, vage. Im BGZ Graz wurde 2000 ein Protokoll zur SNB beim Mammakarzinom mit Spätbild am nächsten Tag eingeführt. Wir untersuchten, ob die szintigraphische Evaluierung am nächsten Tag im Vergleich zu den Frühbildern einen Informationsgewinn brachte.

Material und Methoden: Alle 232 Patientinnen mit Mammakarzinom an der UFK Graz von 10/2013-09/2015, bei denen eine präoperative szintigraphische SN Markierung durchgeführt wurde, wurden retrospektiv ausgewertet. Nach periareolärer Markierung mit 4x37 MBq 99m Tc-Nanokolloid, erfolgte die Szintigraphie 2 h (Frühbild) und 18 h (Spätbild) p.i. Die Ergebnisse der Frühbilder wurden mit den Spätbildern verglichen. Ergebnisse: Bei 232 Patientinnen wurden 238 SN Szintigraphien durchgeführt ( 6 bilaterale Karzinome). In 154,238 Szintigraphien (65\%) war $\geq 1$ SLN im Frühbild darstellbar; bei 84 (35\%) war kein SN darstellbar, davon wurde bei 8,84 kein Spätbild mehr angefertigt. Von den restlichen 76 Fällen kam in 40,76 Fällen (52\%) im Spätbild noch ein SN zur Darstellung, bei 36,76 (48\%) nicht.

Diskussion: Ein darstellbarer LK in der Frühaufnahme nach szintigraphischer Markierung ist definitionsgemäß der SLK. In jenen 35\% der Fälle in unserer Serie, wo im Frühbild kein SLN zur Darstellung kam, war das Spätbild zu 52\% zielführend. 